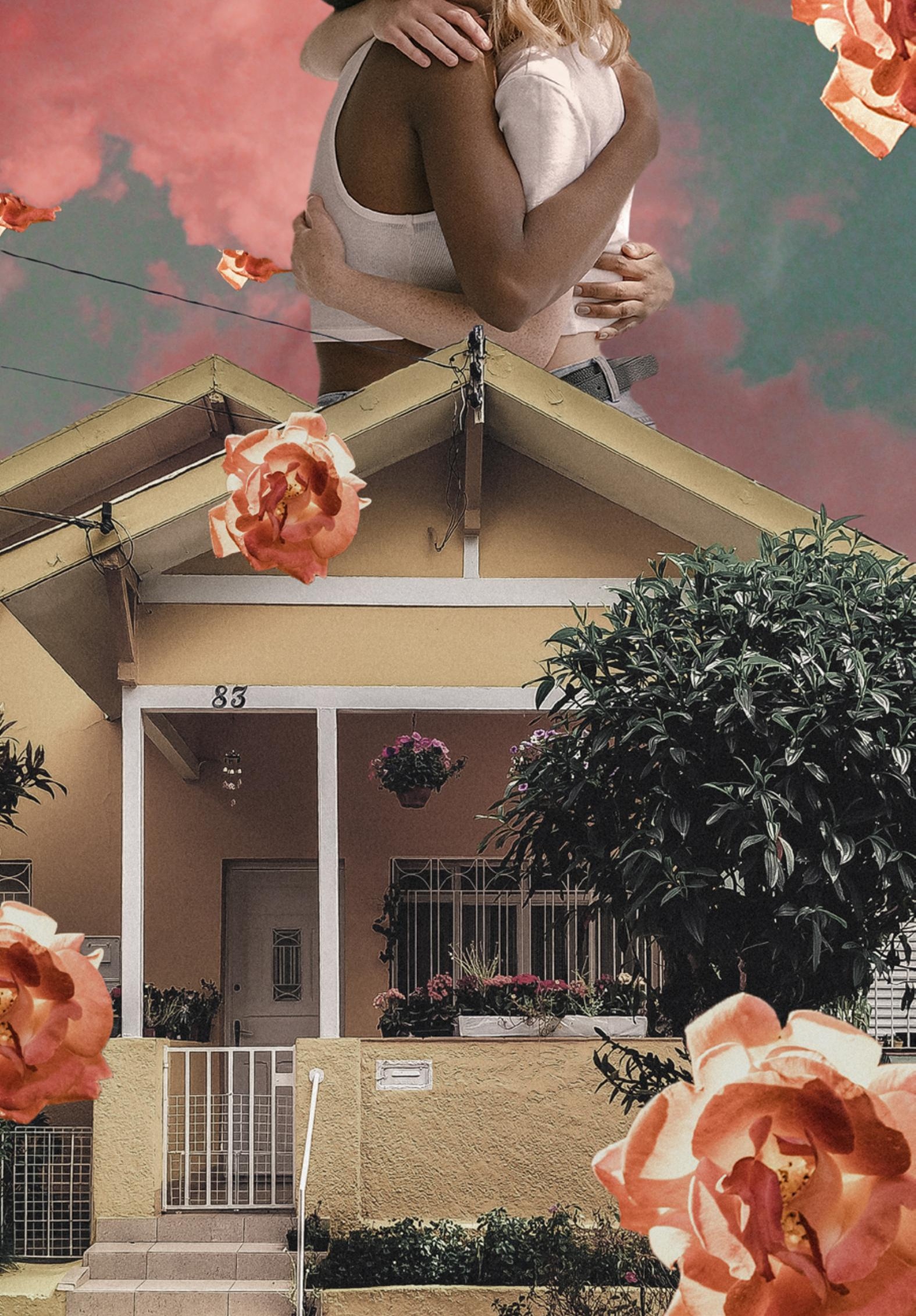




\title{
Vivências e sentimentos compartilhados por usuários de uma casa de apoio a pessoas com câncer
}

Shared experiences and feelings by the users of a support house for cancer patients

\author{
Jarbas da Silva Ziani \\ Graduando em Enfermagem, pela Universidade Federal do Pampa - UNIPAMPA \\ jarbas_ziani@outlook.com \\ Rodrigo de Souza Balk \\ Professor associado pela Universidade Federal do Pampa - UNIPAMPA. \\ rodrigo.balk@gmail.com \\ Carla Gabriela Rodrigues de Souza \\ Graduanda em Fisioterapia, pela Universidade Federal do Pampa - UNIPAMPA \\ carlagrsouza@gmail.com \\ Caroline Andressa Bortoluzzi Zalamena \\ Graduanda em Fisioterapia, pela Universidade Federal do Pampa - UNIPAMPA \\ karol_abz@hotmail.com \\ Mariana Goulart Almiron \\ Fisioterapeuta pela Universidade Federal do Pampa - UNIPAMPA \\ almironmariana26@gmail.com \\ Lalesca Gomes de Oliveira \\ Graduanda em Farmácia, pela Universidade Federal do Pampa - UNIPAMPA \\ lalescagomes@gmail.com
}

\section{RESUMO}

Trata-se de um relato de experiências desenvolvidas por meio de oficinas realizadas por uma equipe multidisciplinar, com o intuito de promover a qualidade de vida de pacientes oncológicos, os quais são amparados por uma casa de apoio. Tais experiências são provenientes de uma atividade desenvolvida por um Programa de Educação Tutorial e Práticas Integradas em Saúde Coletiva de uma Universidade Federal da fronteira oeste do Rio Grande do Sul, que tem como objetivo desenvolver o olhar multidisciplinar dos acadêmicos. A atividade foi desenvolvida em duas oficinas, sendo uma de pilates e outra de dança. Um dos principais achados deste estudo foi identificar a importância das casas de apoio aos pacientes no processo de tratamento contra o câncer, pois muitos olham a casa como um lar temporário que lhes serve de amparo no enfrentamento dessa fase da vida que tanto exige dos pacientes e de seus familiares.

Palavras-chave: Casas de apoio; Oncologia; Qualidade de vida.

\section{ABSTRACT}

It is an account of experiences developed through workshops conducted by a multidisciplinary team in order to promote the quality of life of cancer patients who are supported by a support home. Such experiences come from an activity developed by a Tutorial Education Program and Integrated Practices in Collective Health of a Federal University on the western border of Rio Grande do Sul, which aims to develop the multidisciplinary view of academics. The activity was developed in two workshops, one of pilates and the other one of dance. One of the main findings of this study was to identify the importance of patient support homes in the cancer treatment process, because many see in the house a temporary home that supports them in facing this phase of life that demands so much from patients and their families.

Keywords: Support Houses; Oncology; Quality of Life. 


\section{INTRODUÇÃO}

O diagnóstico de qualquer tipo de neoplasia maligna, usualmente, acarreta a quem o recebe um intenso desgaste emocional ligado, principalmente, aos estigmas que permeiam a doença e às consequências que o tratamento pode vir a provocar no indivíduo, sejam elas relacionadas a fatores econômicos, sejam pela sensação de morte que se faz presente nesse período. Além do mais, esses fatores acabam gerando impactos significativos nas esferas emocional, social e material que esses pacientes enfrentam (Barsaglini \& Soares, 2018).

Diante disso, foi criada em 2005, na cidade de Caxias do Sul-RS, uma Organização da Sociedade Civil (OSC) denominada Associação de Apoio a Pessoas com Câncer (AAPECAN), a qual tem por objetivo atender pessoas que estão vivenciando o tratamento oncológico ou na acolhida de seus familiares que se encontram em situação de fragilidade. Com essa iniciativa, a OSC já está com 14 unidades dispostas por diversos municipios do Rio Grande do Sul.

Essas casas de apoio surgiram com o intuito de promover assistência aos pacientes oncológicos de forma gratuita, uma vez que ofertam um cuidado holístico aos usuários, quebrando o paradigma "hospitalar" do tratamento e visando trazer o conforto necessário em um momento tão doloroso. Assim, com essa iniciativa, propõe-se não só auxiliar os indivíduos com um local, mas também realizar intervenções terapêuticas para um melhor enfrentamento no processo da doença.

A AAPECAN proporciona estada e bem-estar físico e mental em um ambiente acolhedor e de paz para que os usuários tenham os efeitos físicos e mentais desenvolvidos pelo agressivo tratamento minimizados por meio de um acolhimento multidisciplinar, contando com uma escuta especializada (Ferreira, et al., 2015).

O acolhimento ao paciente oncológico oportuniza uma série de mecanismos de defesa ao mesmo tempo que ajuda a superar esse momento. $\mathrm{Na}$ grande maioria dos casos, esse processo se desenvolve também pelo envolvimento emocional por parte do profissional que acompanha essa fase, já que, geralmente, o tratamento é longo e as hospitalizações e os retornos às casas de apoios são frequentes (Luz, et al., 2016).

Assim, com a finalidade de promover o acolhimento do paciente oncológico, o Programa de Educação Tutorial Práticas Integradas em Saúde Coletiva (PET-PISC), que visa ofertar experiências práticas aos acadêmicos da área da saúde, aliou-se à AAPECAN.

Para Biscarde et al., os cursos de graduação, em especial os que envolvem as especialidades da saúde, não devem contemplar apenas o conhecimento técnico-científico, mas também o ético-político, transformando acadêmicos em sujeitos humanistas, de forma a refletir em sociedade conforme as necessidades. Sendo assim, o intuito da experiência é desenvolver um apoio emocional para os pacientes que ali estão, e - do mesmo modo - estimular o pensamento crítico e reflexivo dos acadêmicos, contribuindo com uma formação global e de qualidade. 
Visto isso, o objetivo deste trabalho é descrever as experiências transcorridas por meio de oficinas realizadas por uma equipe multidisciplinar com a finalidade de promover a qualidade de vida de pessoas com diagnóstico de câncer que são amparadas por uma casa de apoio.

\section{METODOLOGIA}

O estudo refere-se a um relato de experiência proveniente de uma atividade desenvolvida pelo grupo PET-PISC, do qual fazem parte bolsistas dos cursos de graduação em Enfermagem, Fisioterapia e Farmácia de uma Universidade Federal da fronteira oeste do Rio Grande do Sul, que tem como objetivo desenvolver o olhar multidisciplinar dos acadêmicos supracitados.

Teve-se como local de prática uma casa de apoio a pessoas com câncer de um município da fronteira oeste do Rio Grande do Sul/Brasil. As atividades foram desenvolvidas de 2018 a 2020, ocorrendo de forma semanal, com a supervisão do tutor dos acadêmicos, da assistente social e da psicóloga da AAPECAN; e a atuação foi dos petianos dos referidos cursos de graduação.

As atividades desenvolvidas no interior da própria AAPECAN apresentam-se como grupos de apoio e são denominados de "Sentimento é arte" e "Esperança". Os nomes dos grupos foram idealizados, a partir do significado que essas palavras possuem para os usuários da casa: "Sentimento é arte" faz uma alusão à aptidão para sentir, e a arte de estar na casa pode fazer com que os usuários enxerguem a vida de diversas formas. Já o grupo "Esperança" se denomina assim, uma vez que essa palavra é vista, por muitos moradores do local, como um sentimento de realização, e todos almejam esse objetivo.

Dessa forma, por meio desses grupos supracitados, eram realizadas oficinas e rodas de conversa, sempre com a condução de uma psicóloga e da assistente social. Durante a parceria firmada entre os dois grupos, o PET-PISC, além de participação nos grupos de apoio, realizava atividades pontuais que ocorriam uma vez por mês, no ambiente da Universidade. Dentre as atividades desenvolvidas, destacam-se as oficinas de Pilates solo e Dança, promovendo a saúde física e mental desses pacientes.

O Pilates solo foi realizado em um laboratório do curso de Fisioterapia, localizado no campus universitário, para onde os pacientes eram encaminhados uma vez por mês. A atividade é composta de exercícios de alongamento, respiração e técnicas de relaxamento, com duração de uma hora, e respeitando as limitações físicas de cada indivíduo, sendo acompanhados pela psicóloga e pela assistente social, tutor e petianos. Após o término da atividade proposta, era oferecido aos pacientes um passeio pelo campus e, na sequência, todos participaram de um lanche coletivo de forma que não fosse alterada a rotina da AAPECAN.

Já a oficina de Dança ocorria nas dependências da AAPECAN com a participação do professor de dança, dos petianos, da assistente social e da psicóloga. Os ritmos eram determinados de acordo com a preferência de todos ali 
presentes, com a duração de uma hora e obedecendo aos desejos e restrições de todos do grupo.

As atividades eram organizadas e planejadas pelos integrantes do grupo PET-PISC em comum acordo com a AAPECAN.

\section{RESULTADOS E DISCUSSÕES}

Atualmente, a casa de apoio a pessoas com câncer conta com 179 cadastros ativos. Desses, 48 residem atualmente na cidade de Uruguaiana, e 131 pertencem aos municipios de Alegrete, São Borja, Santana do Livramento, Dom Pedrito, Itaqui e São Gabriel. A média das idades varia entre 29 a 59 anos. As neoplasias que mais se destacam são câncer de próstata, mama, pulmão, intestino e traqueia.

Por meio da inserção dos bolsistas na AAPECAN, tornou-se nítido, com as vivências com os usuários, que os primeiros sentimentos que eles apresentavam eram de solidão e de insegurança. Em contrapartida, evidenciou-se que, ao longo dos dias, com a inserção deles na rotina da casa, os sentimentos de acolhimento e de pertencimento ganharam destaque, seja pela relação de afeto com os profissionais da casa de apoio, seja pelos petianos; e por meio desse vínculo criado, eles conseguem, muitas vezes, se desligar dos anseios que tanto lhes afligiam antes de chegar à casa; e assim, passam a manter uma perspectiva positiva de sua doença. É perceptivel que a assistência integral nesse periodo permite que eles venham a ter uma melhor qualidade de vida, pois esses fatores influenciam diretamente no bem-estar físico e psicossocial (Calil \& Prado, 2009).

Do mesmo modo, amenizando os efeitos advindos das fases do tratamento, aconselha-se os individuos a participarem ativamente dos grupos que são ofertados, visto que eles promovem apoio emocional que beneficia diretamente, com a melhoria da percepção, a sensibilidade do próprio corpo, a consciência corporal e a diminuição do nível de ansiedade, aliviando os efeitos do estresse, como hipertensão, úlceras, indigestão, problemas infecciosos (Fontes, et al., 2019).

Assim, o grupo desenvolveu oficinas de promoção à saúde dos usuários as quais serão descritas na sequência deste estudo.

\section{OFICINA DE PILATES}

\section{"Compartilhando emoções"}

O método pilates foi idealizado por Joseph Hubert Pilates durante o século XX, e começou a ser disseminado após sua morte, em 1967. Essa prática é baseada em principios para que os movimentos proporcionem concentração, respiração, centralização, fluidez, controle e precisão. Os exercicios podem ser 
executados tanto em aparelhos elaborados, quanto no chão, o que se denomina de mat pilates. (Juliano \& Bernardes, 2003).

O desenvolvimento da atividade foi precedido por uma reunião entre a AAPECAN e o grupo PET-PISC, com a finalidade de pactuar a oficina e considerar todos os aspectos que permeiam os usuários. Logo, o grupo começou a idealização da atividade, que foi intitulada "Compartilhando emoções" e contou com a participação de 6 acadêmicos, sendo 3 do curso de graduação em Fisioterapia, 3 de Enfermagem e mais a participação do tutor. Além desses, contouse com as participações da assistente social e da psicóloga da instituição.

As técnicas de pilates propostas foram ministradas pelas bolsistas de Fisioterapia com a supervisão do tutor. Os demais prestaram auxilio e monitoramento para evitar algum prejuizo à sua saúde. Quanto ao número de participantes, a atividade contou com 21 usuários, sendo 13 do sexo feminino, com média de idades entre 36 e 59 anos. Ao adentrar na sala, todos os usuários passaram por uma triagem de sinais vitais e pela realização de uma anamnese para que pudéssemos ter certeza se todos estavam realmente aptos a participar da atividade.

O método pilates tem como sua essência a coordenação do corpo, mente e espírito, criado com a proposta de aprimorar o corpo por meio da correção da postura, restabelecendo o ânimo de vida e proporcionando uma melhor qualidade de vida refletida na mente e no espírito do individuo, tornando-se uma ferramenta para a reabilitação de pessoas que demandam de uma assistência complexa, como é situação de pacientes oncológicos (Cruz, et al., 2016).

A atividade física indicada com devida responsabilidade para o paciente oncológico tem uma importância durante ou após o tratamento, sendo uma influência no prognóstico, impactando na deficiência cardiorrespiratória, fadiga relacionada ao câncer, o linfedema, principalmente a qualidade de vida (Eickmeyer, et al., 2012).

Os exercícios de pilates, associados com exercícios respiratórios, de força e de alongamentos, mostraram-se favoráveis na recuperação e na qualidade de vida de mulheres após cirurgia de câncer de mama. O método Pilates não é superior a algum exercício, mas apresenta ganhos aos seus praticantes, principalmente no tocante à qualidade de vida, tornando-se um recurso para o acréscimo de melhora no tratamento (Odynets, et al.,2019).

A prática de exercício físico em grupo foi um fator crucial para que os usuários se sentissem confortáveis em realizar as atividades propostas, bem como para gerar um ambiente descontraído e afetuoso, no qual se perceberam os vínculos criados entre os pacientes e a equipe de assistência à pessoa com câncer. É importante ressaltar que os exercícios foram adaptados para que todos pudessem realizar dentro de suas particularidades, tendo como compromisso manter os princípios do método Pilates dentro dos exercícios realizados.

Quanto à infraestrutura, o grupo preocupou-se em oferecer todas as medidas de segurança. Por isso, optou-se por realizar as atividades nas dependências dos laboratórios do curso de Fisioterapia da Universidade Federal do Pampa-UNIPAMPA, a qual possui os materiais para a prática de pilates. Os 
exercícios realizados foram: Alongamento de Cadeia Posterior em pé 3x de 30 segundos. Os exercícios de Mat Pilates foram: Ponte, Roll Over, Spine Strach, Saw, realizados em 3 séries de 7 vezes, com intervalo de um minuto de descanso entre cada série, ao final foi realizada uma dinâmica com música e com a bola suiça média de $55 \mathrm{~cm}$, em que foi associada a atividade de passar a bola a uma atividade cognitiva, como lembrar os utensilios de algum cômodo da casa. Foram utilizados os seguintes materiais: Colchonetes (12), Faixa Elástica (12), Bola Suiça (1).

Ademais, inferiu-se, com essa Oficina, que a prática de Pilates foi de extrema importância para eles, o que ficou evidenciado por meio de relatos. Além disso, é notória a ajuda no processo de reabilitação desses pacientes, uma vez que provocou neles uma melhora na saúde mental e física. Nesse contexto, ressalta-se que o pilates pode ser utilizado em grande parte da população, uma vez que permitiu inúmeros benefícios aos seus pacientes e possuem poucas contraindicações para o tratamento de pacientes oncológicos (Barbara, et al., 2018).

Figura 1
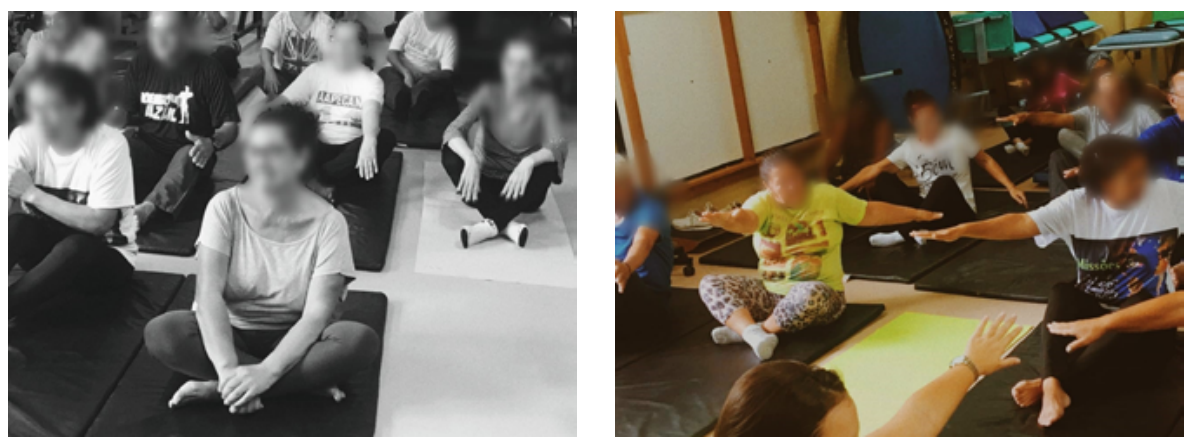

As figuras 1 e 2 apresentam, respectivamente, a orientação e a execução da atividade de Pilates.

\section{OFICINA DE DANÇA}

\section{"Dançar é clamar por felicidade"}

Para a realização da atividade, contou-se com a participação de 5 acadêmicos, 3 do curso de graduação em Enfermagem e 2 do curso de Fisioterapia, além da assistente social e da psicóloga da AAPECAN, e do professor de dança. Dessa atividade, participaram 16 usuários, sendo 10 do sexo feminino, com média de idade dos participantes entre 45 e 58 anos. A atividade foi desenvolvida nas dependências da AAPECAN, em uma área externa disponivel para 
a realização de atividades. Já as coreografias deram-se por ritmos variados, desde o funk às músicas tradicionalistas da região.

Ao término da atividade, foi solicitado aos usuários que eles realizassem um breve feedback sobre a atividade proposta. E, após esses relatos, identificou-se, por meio da alegria e da motivação que eles expressaram, que a atividade cumpriu com o esperado, tornando-se gratificante ouvir as manifestações de agradecimento dos pacientes, pois foram unânimes os relatos de que a oficina foi empoderadora. Desse modo, os estudos já comprovam que o ato de dançar contribui significativamente na reabilitação do paciente (Marbá, Silva \& Guimarães, 2016).

Figura 3

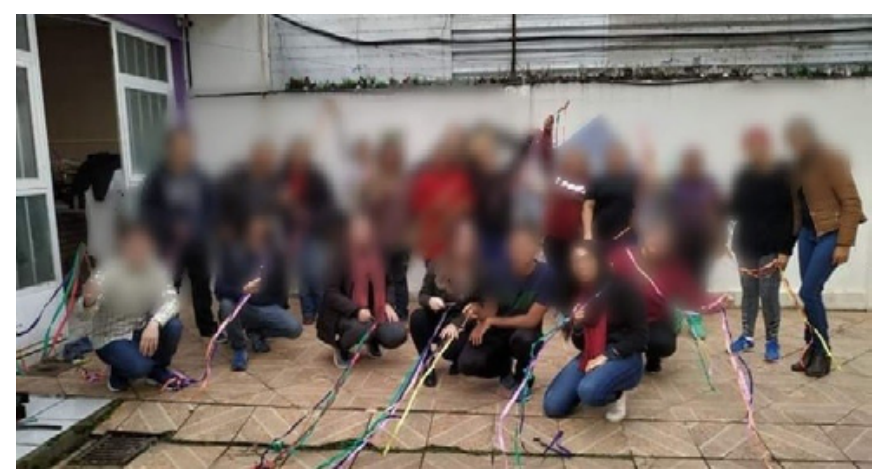

\section{AÇÃO}

\section{"Dançar é clamar por felicidade"}

Por intermédio das atividades citadas, pôde-se observar os impactos positivos no que repercute a visão multiprofissional aos pacientes, uma vez que a participação efetiva desses profissionais garante uma troca de conhecimento, promovendo experiências compartilhadas e maior autocuidado.

Além das práticas mencionadas, também se busca encontrar meios de dispor de uma fonte de renda por intermédio de artesanatos, costura entre outras condutas que são executadas dentro da AAPECAN. Ações como as descritas nesse estudo corroboram com resultados de pesquisas já realizadas no que diz respeito à relação multidisciplinar ser vista como uma parceira na resolubilidade das complicações de saúde, uma vez que ela, sendo bem empregada, tem como objetivo promover autonomia e segurança ao usuário (Silva, Ferreira \& Carneiro, 2017). 
Tem-se observado que o método dinâmico de ensino e aprendizagem que é ofertado pelo programa dentro da AAPECAN, possibilita um olhar mais abrangente dos bolsistas quanto ao processo de adoecer, visto que ele engloba diversas fases da vida do sujeito, favorecendo que os petianos se desprendam do padrão biomédico que ainda é muito empregado nas disciplinas da graduação e os direcione a ver a pessoa que existe além daquela doença. Com isso, promovem-se experiências produtivas e agregadoras para o coletivo pelo interior do olhar multidisciplinar do grupo.

Além do mais, no decorrer desses momentos, é possivel mensurar o quanto essas atividades proporcionam beneficios, pois é por meio delas que os petianos adquirem novos conhecimentos, ganhando responsabilidades, sensibilidade com o próximo, amadurecimento e comprometimento para saírem da Universidade aptos para intervir consideravelmente na sociedade. Os envolvidos nas experiências foram capazes de compreender que, ao longo das discussões, a vivência na AAPECAN leva a momentos de satisfação, crescimento pessoal e prazer, minimizando alguns momentos de desgastes e sofrimentos antes sentidos, uma vez que a situação que se evidencia na casa é digna de uma reflexão sobre aquilo que estamos sentindo e que merece tanta relevância.

Ressaltava-se que as atividades foram registradas com fotos, filmagens e relatos, pois os registros facilitam o planejamento diário, a sistematização de novas ações, a avaliação do processo, bem como a socialização com os usuários.

\section{CONSIDERAÇÕES FINAIS}

Destarte, salienta-se a importância das casas de apoio aos pacientes no processo de tratamento contra o câncer, pois muitos veem na casa um lar temporário que thes serve de amparo no enfrentamento dessa fase de vida que exige tanto dos pacientes e de seus familiares. Ademais, nessa óptica, é imprescindivel ver o paciente oncológico como um ser holístico, compreendendo todas as suas necessidades para o tratamento de forma ativa e saudável para minimizar tanto os agravos psicológicos, quanto os físicos que eles possam vir a enfrentar.

Portanto, após a participação nos grupos, os autores puderam vivenciar experiências excepcionais que contribuíram significativamente para as suas sensibilizações enquanto futuros profissionais e também na construção de seus pensamentos críticos e reflexivos, uma vez que, por meio dos relatos, tornouse evidente que a acolhida do programa PET-PISC influencia na permanência no processo de tratamento e de aceitação da doença pelo usuários, resultado das intervenções em grupo que oferecem a possibilidade de um modelo que pode ser adaptado a diversas situações, assim contribuindo para a segurança, autonomia e qualidade de vida do usuário. 


\section{REFERÊNCIAS}

Barsaglini, Reni Aparecida, \& Soares, Beluci Bianca Nunes de Siqueira. (2018). Impactos de adoecimento de longa duração: experiência de adultos jovens com Leucemia Mieloide Aguda. Ciência \& Saúde Coletiva, 23(2), 399-408. https://dx.doi.org/10.1590/1413-81232018232.15442017

Barbara, Jussara Luiza, Lima, Barbara Benevides, Sales, Laura Boechat Pires Almeida, Teixeira, Pâmela Rangel, Baracat, Patrícia Junqueira Ferraz; Soares, Mairkon Almeida. (2018). O efeito do método pilates sobre o recrutamento de unidades motoras e flexibilidade em pacientes mastectomizadas. Perspectivas Online: Biológicas \& Saúde, 8(28), 24-41. http://dx..doi.org/10.25242/886882820 181425

Biscarde Daniela Gomes dos Santos, Pereira-Santos Marcos, Silva Lília Bittencourt. (2014). Formação em saúde, extensão universitária e Sistema Único de Saúde (SUS): conexões necessárias entre conhecimento e intervenção centradas na realidade e repercussões no processo formativo. Interface (Botucatu), 18(48), 177-186. http://dx.doi.org/10.1590/1807-57622013.0586

Calil, Ana Maria, \& Prado, Cláudia. (2009). O ensino de oncologia na formação do enfermeiro. Revista Brasileira de Enfermagem, 62(3), 467-470. https://doi. org/10.1590/So034-71672009000300022

Cruz, Josiane Cristiane, Liberali, Rafaela, Cruz, Ticiane Marcondes Fonseca da, \& Netto, Maria Ines Artaxo. (2016). The Pilates method in the rehabilitation of musculoskeletal disorders: a systematic review. Fisioterapia em Movimento, 29(3), 6og-622. https://doi.org/10.1590/1980-5918.029.003.AO19

Eyckmeyer, Sara; Gamble, Gail; Shahpar,Samman; Kim,Do.(2019). The Role and Efficacy of Exercise in Persons With Cancer. Function and Rehabilitation,Theme Issue, Exercise and Sports for Health Promotion Disease, And Disability. PM\&R -The Journal of Injury, 4, (11), 874-881. https://doi.org/10.1016/j.pmrj.2012.09.588

Ferreira, Patrícia Chatalov, Wakiuchi, Julia, Baldissera Vanessa Denardi Antoniassi, Sales Catarina Aparecida. (2015). Sentimentos existenciais expressos por usuários da casa de apoio para pessoas com câncer. ESC. Anna Nery, 19(1), 66-72. http://dx.doi.org/10.5935/1414-8145.20150009

Fontes, A., de Araújo Patrício, A., de Lima, L., dos Santos, T., do Nascimento, L., \& Rosendo da Silva, R. (2019). Stress vulnerability: parents that take care of cancer bearing children / Vulnerabilidade ao estresse: pais cuidadores de filhos com câncer. Revista de Pesquisa: Cuidado é Fundamental Online, 11(4), 857-861. http://dx.doi.org/10.9789/2175-5361.2019.v11i4.857-861 
Juliano, Rafael de Arruda; Bernardes, Rodrigo. (2016). A Teoria do Método Pilates: Da História à Biomecânica.(2a ed).Porto Alegre: Ideograf.

Luz Kely Regina da, Vargas Mara Ambrosina de Oliveira, Barlem Edison Luiz Devos, Schmitt Pablo Henrique, Ramos Flávia Regina Souza, Meirelles Betina Hörner Schlindwein. (2016). Estratégias de enfrentamento por enfermeiros da oncologia na alta complexidade. Rev. Bras. Enferm. 69(1), 67-71. https://doi.or$\mathrm{g} / 10.1590 / 0034-7167.2016690109 i$

Marbá, Romolo Falcão, Silva, Geusiane Soares, Guimarães, Thamara Barbosa. (2016). Dança na promoção da saúde e melhoria da qualidade de vida. Revista Científica do ITPAC, Araguaina, 9(1): 1:9. https://www.redalyc.org/jatsRepo/4979/497950366008/html/index.html

Odynets, Tetiana; Briskin,Yuriy;Todorova,Valentina. (2019). Effects of Different Exercise Interventions on Quality of Life in Breast Cancer Patients: A Randomized Controlled Trial.Journal List, Integrative Cancer Therapies, 18, 1-8. https:// doi.org/10.1177/1534735419880598

Silva, Natália Ferreira; Ferreira, Natália Madureira, Carneiro, Nicole Geovana Dias. (2017). Ação multidisciplinar na educação permanente de agentes comunitárias de saúde. Rev. Ed. Popular, Uberlândia, 16(3), 156-165. http://www.seer. ufu.br/index.php/reveducpop/article/view/40878/pdf.

Data de submissão: 10/02/2020

Data de aceite: 06/08/2020 